\title{
Pulmonary Hyperinfection with Strongyloides stercoralis in an Immunocompetent Patient
}

\author{
Sadik Mohammed ${ }^{1}$, Pradeep Bhatia ${ }^{2}$, Swati Chhabra ${ }^{3}$, Sunit Kumar Gupta ${ }^{4}$, Rakesh Kumar ${ }^{5}$
}

\begin{abstract}
Strongyloids stercoralis is a unique parasite as it has the capability of completing its life cycle entirely within the human host. The immune system of the host plays an important role in keeping the infection under control but when there is a breach in this system the infection may flare up and leads to hyperinfection. In immunocompetent patients, gastrointestinal manipulation could be an inciting trigger leading to translocation of larva into the systemic circulation and development of hyperinfection syndrome. We report a case where infection with $\mathrm{S}$. stercoralis lead to hyperinfection in patient with intact immune system following laparotomy.

Keywords: Hyperinfection syndrome, Laparotomy, Pulmonary strongyloidosis, Radiology, Sputum examination

Indian Journal of Critical Care Medicine (2019): 10.5005/jp-journals-10071-23267
\end{abstract}

\section{INTRODUCTION}

Strongyloidosis is a helminthic infection caused by Strongyloides stercoralis. Its unique characteristic of completing its life cycle entirely within the human host enables it to cause autoinfection. Respiratory manifestations in an immunocompetent person include pulmonary infiltrates with eosinophilia syndrome or asthma only, while in an immunocompromised patient, the hyperinfection could be life-threatening, with lung abscess, cavitary lung disease, fibrosis and acute respiratory failure.

This case highlights the unusual presentation and diagnostic and management challenges of $S$. stercoralis hyperinfection in a patient with an intact immune system.

\section{Case Description}

A 21-year-old male, farmer by occupation, presented to the emergency department with abdominal pain and vomiting for the last 3 days. The colicky pain was of moderate-to-severe degree and was situated around the umbilical region. It was associated with vomiting (greenish in colour, 8-10 episodes/day) and constipation. The patient was averagely built, poorly nourished and had stable vitals. His abdomen was distended and resonant on percussion without shifting dullness and fluid thrill. The routine investigations were unremarkable except leukocytosis (total leukocyte count 15,850/ $\mu \mathrm{L} ; \mathrm{N}-78.4 \% / \mathrm{L}-15 \% / \mathrm{B}-2.9 \% / \mathrm{E}-3.4 \%)$. Contrast-enhanced computed tomography (CECT) of the abdomen showed "small bowel low grade subacute intestinal obstruction with small bowel feces sign along with mild ileal wall thickening, mild ascites and reactive mesenteric lymphadenopathy". With a provisional diagnosis of subacute intestinal obstruction the patient was scheduled for exploratory laparotomy which revealed inflamed mesentery without any cause of obstruction. The surgery was uneventful and after recovery from general anesthesia the patient was shifted to ward.

After 48 hours in the postoperative ward, the patient developed cough and breathlessness, became dyspnoeic and tachypneic, with diffuse bibasilar crepitation. The oxygen saturation dropped to $78 \%$ breathing room air. Oxygen supplementation was started and the patient was shifted to ICU. Antibiotic was escalated to piperacillin
${ }^{1-5}$ Department of Anesthesiology and Critical Care, All India Institute of Medical Sciences, Jodhpur, Rajasthan, India

Corresponding Author: Sadik Mohammed, Department of Anesthesiology and Critical Care, All India Institute of Medical Sciences, Jodhpur, Rajasthan, India, Phone: +91-9414849733, e-mail: drmsadik@ gmail.com

How to cite this article: Mohammed S, Bhatia P, Chhabra S, Gupta SK, Kumar R. Pulmonary Hyperinfection with Strongyloides stercoralis in an Immunocompetent Patient. Indian J Crit Care Med 2019;23(10):481483.

Source of support: Nil

Conflict of interest: None

and tazobactam after sending samples (blood, sputum and urine) for culture and sensitivity. Chest roentgenogram, which was normal preoperatively, revealed multiple miliary shadows (Fig. 1). By the evening, the patient had two episodes of hemoptysis and worsening of respiratory distress and oxygen saturation. There were diffuse crepitations all over the lung fields. In view of deterioration of his clinical condition, he was intubated and put on mechanical ventilation. Following intubation the endotracheal tube was filled with frothy blood. The $\mathrm{FiO}_{2}$ was increased to 1.0 and PEEP to $15 \mathrm{~mm} \mathrm{Hg}$ to have $\mathrm{PaO}_{2}$ of $63 \mathrm{~mm} \mathrm{Hg}$. Next day, the sputum examination showed the presence of filariform larva of S. stercoralis. Anthelminthic medication (albendazole $400 \mathrm{mg}$ BD and ivermectin $6 \mathrm{mg} \mathrm{OD)}$ was initiated. Intravenous tranexamic acid $500 \mathrm{mg}$ was started in view of alveolar hemorrhage. From the next two days onward, bleeding from tube reduced significantly and ventilator parameters $\left(\mathrm{FiO}_{2}-0.6, \mathrm{PEEP}-12\right.$ to have $\mathrm{PaO}_{2}$ of 72 $\mathrm{mm} \mathrm{Hg}$ ) also improved. Stool sample also confirmed the presence of larva of S. stercoralis. CECT thorax showed consolidation in superior and posterior basal segments of the right lower lobe, interlobular thickening, diffuse miliary opacities and ground glass density nodules in the bilateral lung fields with apicobasal and anteroposterior gradient (Fig. 2).

Repeat hemogram revealed eosinophil counts $10 \%$ and hemoglobin $6.4 \mathrm{~g} / \mathrm{dL}$ for which two units of PRBCs were transfused. Over the next few days, the patient gradually improved on the

() The Author(s). 2019 Open Access This article is distributed under the terms of the Creative Commons Attribution 4.0 International License (https://creativecommons. org/licenses/by-nc/4.0/), which permits unrestricted use, distribution, and non-commercial reproduction in any medium, provided you give appropriate credit to the original author(s) and the source, provide a link to the Creative Commons license, and indicate if changes were made. The Creative Commons Public Domain Dedication waiver (http://creativecommons.org/publicdomain/zero/1.0/) applies to the data made available in this article, unless otherwise stated. 

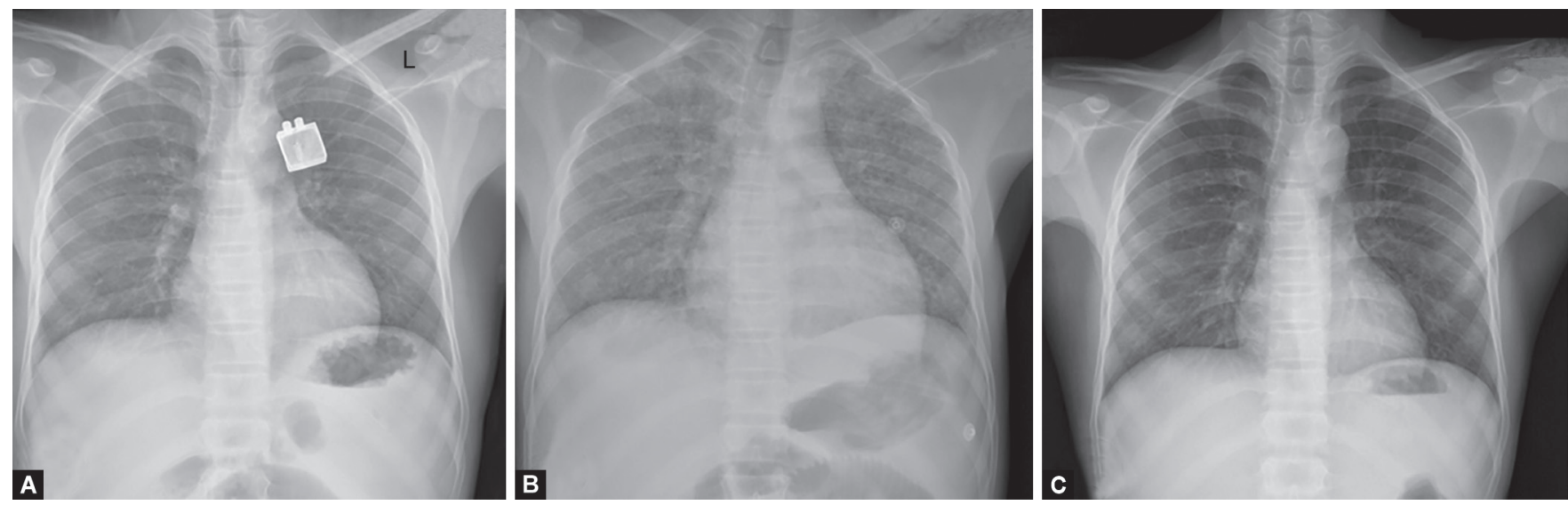

Figs $1 \mathrm{~A}$ to $\mathrm{C}$ : Chest X-ray PA views. (A) At admission showing no obvious abnormality; (B) After admission to ICU showing bilateral diffuse miliary shadow; (C) After recovery showing clearance of miliary shadow
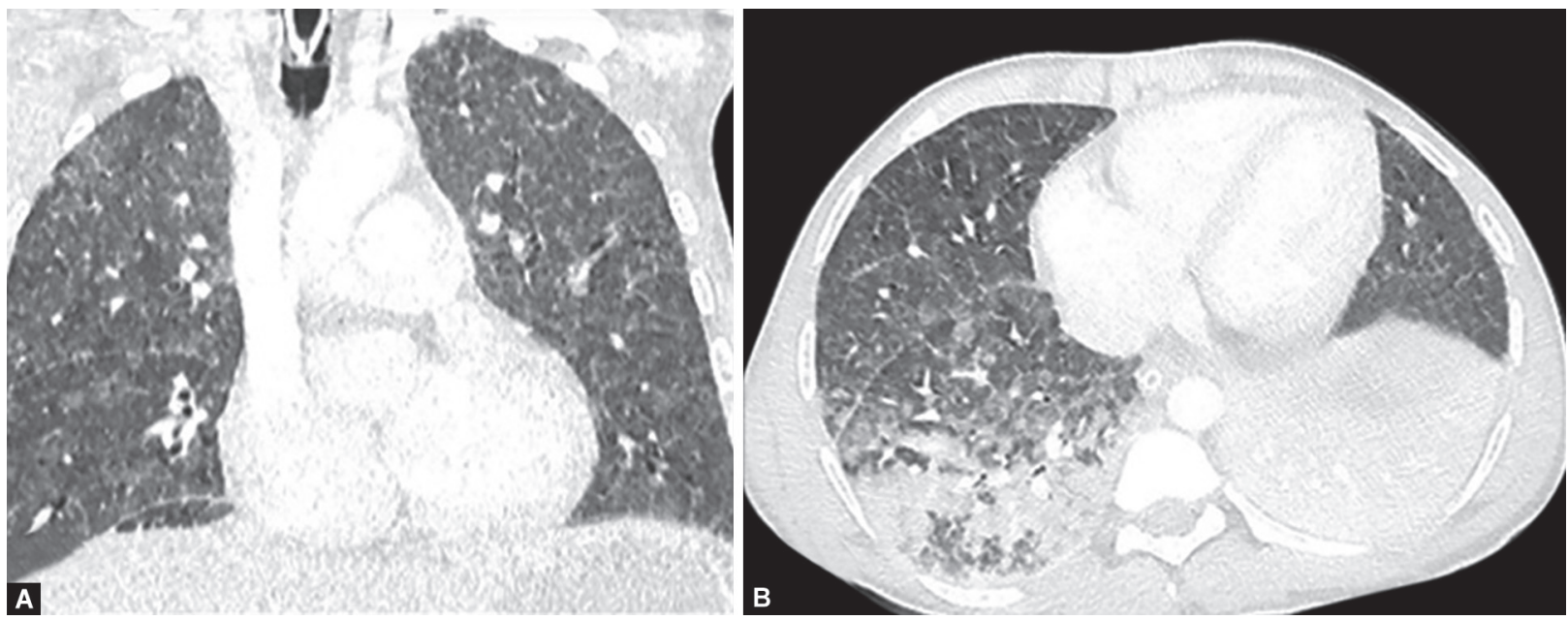

Figs 2A and B: Contrast-enhanced computed tomography of the chest. (A) Right lower lobe consolidation and ground-glass opacities, more posteriorly than in anterior lung field; (B) Military nodules and ground-glass opacities distributed throughout both lungs

ventilator with improvement in radiological findings (Fig 1C). After gradual weaning, the patient was extubated and antibiotic was stopped as there was no evidence of infection (leukocytosis, fever etc). On day 10, ivermectin was discontinued and the patient was shifted to the ward. On day 12, the sputum sample was negative for the parasite and the patient was discharged to home. The patient was advised to continue albendazole $400 \mathrm{mg} \mathrm{BD}$ for one more week. On follow up albendazole was discontinued when sputum sample again was negative for the larvae.

\section{Discussion}

Strongyloidiasis is an infection caused by S. stercoralis, a helminth present mainly in tropical and subtropical regions but also in temperate climates and affect around 100 million individuals worldwide. ${ }^{2,3}$ Its unique and unusual life cycle displays many distinct features that have important implications. Parthenogenesis (female generates the progeny even without copulation) provides the species a survival advantage. Autoinfection (noninfectious larvae mature directly into the infectious larvae while still in the human intestine) allows the infection to continue in the host for many years. ${ }^{4}$ Although the host's immune mechanisms can keep the autoinfection under control, it cannot eradicate it. Consequently, infection continues at a low worm burden to the host. As this control is lost in situations such as immunosuppression, accelerated autoinfection can lead to hyperinfection (high worm load) and dissemination. ${ }^{5}$ Hyperinfection, usually restricted to the organ of autoinfective cycle, is characterized by enhanced GI and pulmonary symptoms with increased numbers of larvae seen in stool or sputum. The term dissemination implies migration of larvae to the organs other than $\mathrm{Gl}$ and pulmonary organs. The mortality rate of hyperinfection in and immunosuppressed individual was reported to be as high as $86 \%{ }^{6}$ The present case was unusual as detailed inquiry related to the immune system (corticosteroid therapy, immunosuppressive drug therapy, HIV/AIDS, malignancy etc.) of the patient revealed intact immunity.

The lung is the most common extraintestinal organ involved in hyperinfection and the diagnosis requires a high index of suspicion as clinical signs and symptoms and radiographic findings are largely nonspecific. ${ }^{7}$ In patients with autoinfection, the migration of larvae from capillary beds into the alveoli produces a foreign body reaction, inflammatory pneumonitis, and pulmonary hemorrhage. During the initial phases of the infection the chest radiograph and CT scan show fine miliary nodules or diffuse reticular infiltrates. As the infection progresses, patchy and diffuse lobar infiltrates can develop. In patients with preexisting lung disease, massive larval migration through the lungs can lead to extensive lung infiltration and development of ARDS. ${ }^{7,8}$ 
Chan et al. ${ }^{9}$ reviewed nine published cases of hyperinfection in immunocompetent patients. They found that male patients with a median age of 48 years were most affected. Patients commonly presented with abdominal pain, fever and diarrhea and were found to have eosinophilia and pulmonary infiltrates on radiological imaging. The diagnosis was made through stool microscopy, from bowel or bronchial biopsies, by sputum microscopy or by the combination of tests. Treatment varied between cases and included ivermectin, albendazole, thiabendazole or a combination. Pulmonary complications ranging from lung abscess to ARDS occurred in five cases, and three patients out of ten died (including the one reported by the authors).

Our patient presented with sign and symptoms suggestive of intestinal obstruction for which he underwent laparotomy. Similarly, the patient reported by Chan et al. underwent laparotomy for intestinal obstruction after which he developed hyperinfection syndrome. In another case report by Myint et al., the patient developed symptoms of hyperinfection following esophagoduodenoscopy and biopsy. We hypothesize that bowel handling in all the above cases could have led to the migration of a large number of $S$. stercoralis larva into the systemic circulation and development of hyperinfection syndrome in the immediate postoperative period. . $^{9} 10$

In the present case hemoptysis and miliary involvement of lungs initially raised the suspicion of tubercular infection which subsequently was found to be caused by S. stercoralis. The patient developed alveolar hemorrhage and ARDS requiring high ventilatory support. He responded well to combination anthelminthic therapy consisting of albendazole and ivermectin, broad-spectrum antibiotic and intravenous fibrinolytic.

\section{Conclusion}

Hyperinfection with S. stercoralis, although common in immunocompromised patients, may also affect immunocompetent patients and require a high index of suspicion for diagnosis in view of nonspecific sign and symptoms. In these patients gastrointestinal manipulation could be an inciting trigger leading to translocation of larva into the systemic circulation and development of hyperinfection syndrome. Aggressive management in an intensive care unit including anthelminthic medication, mechanical ventilation and broad-spectrum antibiotic early in the course of the disease may result in favorable outcome.

\section{References}

1. Mokhlesi B, Shulzhenko O, Garimella PS, Kuma L, Monti C. Pulmonary Strongyloidiasis: The Varied Clinical Presentations. Clin Pulm Med. 2004;11(1):6.

2. Genta RM. Global prevalence of strongyloidiasis: critical review with epidemiologic insights into the prevention of disseminated disease. Rev Infect Dis 1989;11:755-767.

3. Segarra-Newnham M. Manifestations, diagnosis and treatment of Strongyloides stercoralis infection. Ann Pharmacother. 2007;41(12):1992-2001.

4. Liu LX, Weller PF. Strongyloidiasis and other intestinal nematode infections. Infect Dis Clin North Am 1993;7:655-682.

5. Grove DI. Human strongyloidiasis. Adv Parasitol 1996;38:251-309.

6. Igra-Siegman Y, Kapila R, Sen P, Kaminski ZC, Louria DB. Syndrome of hyperinfection with Strongyloides stercoralis. Oxford J Med Clin Infec Dis. 1981;3:397- 407.

7. Woodring JH, Halfhill H 2nd, Berger R, Reed JC, Moser N. Clinical and imaging features of pulmonary strongyloidiasis. South Med J 1996;89:10-19.

8. Woodring JH, Halfhill H, Reed JC. Pulmonary strongyloidiasis: clinical and imaging features. Am J Roentgenol 1994;162:537-542.

9. Chan FLY, Kennedy B, Nelson R. Fatal Strongyloides hyperinfection syndrome in an immunocompetent adult with review of the literature. Internal Medicine Journal 2018;48:872-875.

10. Myint A, Chapman C, Almira-Suarez I, Mehta N. Strongyloides hyperinfection syndrome in an immunocompetent host resulting in bandemia and death. BMJ Case Rep 2017. 